\title{
AN EXTRAORDINARY VIEW OF THE UNIVERSE
}

\author{
THE USE OF X-RAY VISION IN SPACE SCIENCE
}

\section{ANETA SIEMIGINOWSKA}

X-ray emission from cosmic sources indicates that these sources are heated to temperatures exceeding a million degrees or that they contain highly energetic particles. Recent X-ray telescopes, such as the Chandra X-ray Observatory and XMM-Newton, observed thousands of cosmic X-ray sources. These observations greatly impacted our understanding of the physics governing the evolution of structures across the universe. Here, I review and highlight some of these important results.

Keywords: astronomy, X-rays, quasars, jets, X-ray clusters, cosmology.

Riccardo Giacconi received the 2002 Nobel Prize in Physics «for pioneering contributions to astrophysics, which have led to the discovery of cosmic X-ray sources». The final paragraph of his Nobel Lecture, held on December 8, 2002, summarized the importance of X-ray astronomy:

... this radiation reveals the existence of astrophysical processes where matter has been heated to temperatures of millions of degrees or in which particles have been accelerated to relativistic energies. The X-ray photons are particularly suited to study these processes because they are numerous, because they penetrate cosmological distances, and because they can be focused by special telescopes. This last property significantly distinguishes X-ray from gammaray astronomy. However, in a more fundamental way, high energy astronomy has great importance in the study of the universe because high energy phenomena play a crucial role in the dynamics of the universe.

(Giacconi, 2002).

In 1960, Riccardo Giacconi and Bruno Rossi published a short article titled «A "telescope" for soft $\mathrm{X}$-ray astronomy, which described the theory and design of an X-ray telescope. The Earth's atmosphere is opaque to $\mathrm{X}$-ray radiation so observations of $\mathrm{X}$-ray sources require telescopes located in space.
In addition, the wavelength of X-ray radiation is shorter than 10 Angstroms and focusing these very short electromagnetic waves involves their reflection at very small angles (grazing) off the surface of the telescope's mirror. The X-ray telescope presented by Giacconi and Rossi would focus the X-rays and give sharp images of the X-ray sky, which was not known at that time.

In the same year Giacconi, George W. Clark, and Rossi published predictions of X-ray fluxes from a few sources outside the solar system. Their predictions indicated that studies of extragalactic X-ray sources needed very sensitive and large telescopes, new types of detectors and new data analysis methods. These early theoretical ideas initiated investigations of the X-ray universe. Since then, many X-ray missions collected data showing that violent events are common and the universe is not calm at all. One important quality of all X-ray missions was an open access to X-ray observations. Open access to data archives, with common data formats, analysis software, availability of raw data and source catalogs is still a particular quality of X-ray research. NASA's High Energy Astrophysics Science Archive Research Center (HEASARC) web page ${ }^{1}$ contains details of

\footnotetext{
1 https://heasarc.gsfc.nasa.gov/
} 


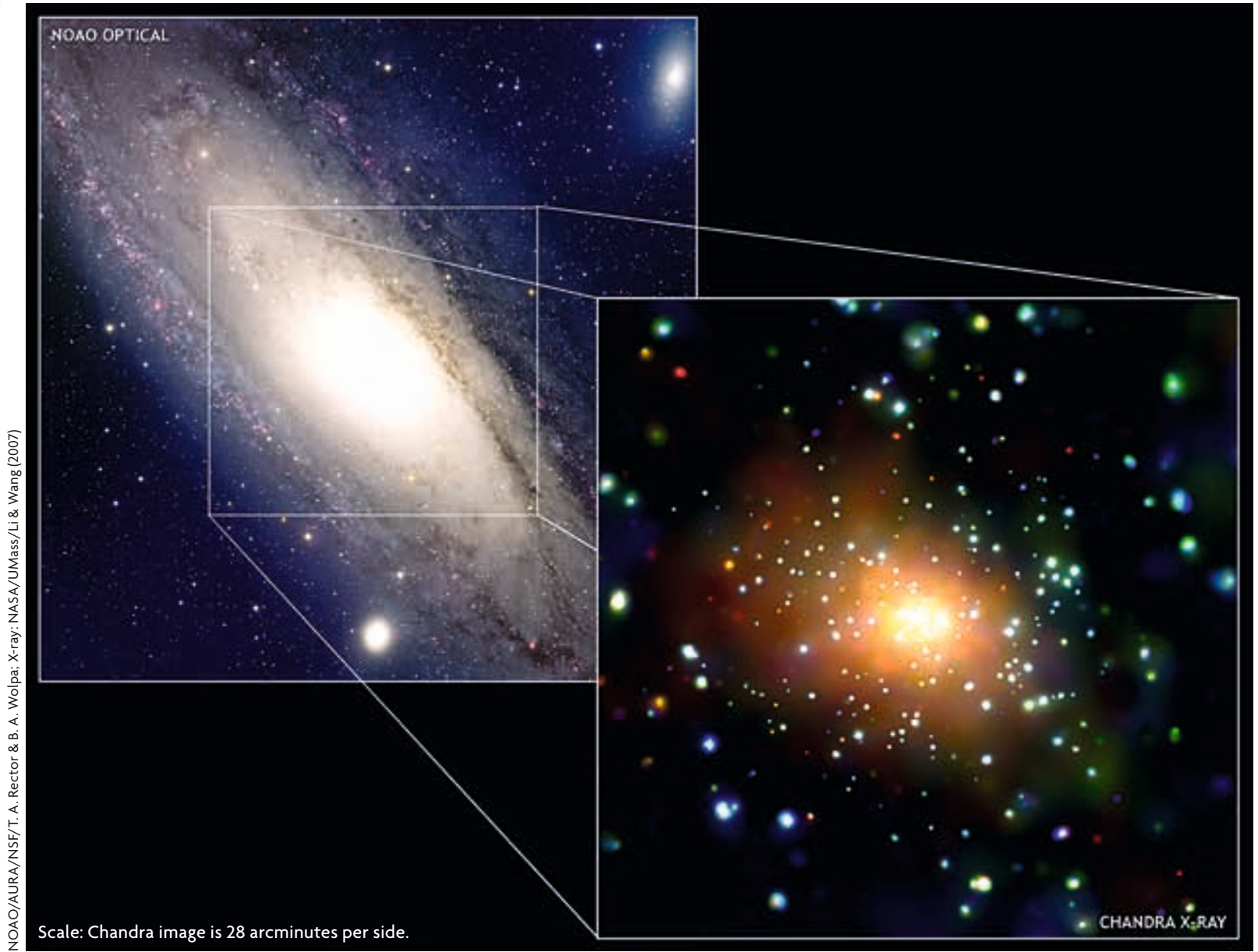

Figure 1. National Optical Astronomy Observatory (NOAO) optical image (left) \& Chandra X-ray image (right) of Andromeda Galaxy, also known as M31. Located in the constellation Andromeda («the Princess»), M31 is a large spiral galaxy very similar to the Milky Way. The Chandra X-ray image shows the center of the Andromeda Galaxy in the three bands (red, low energy; green, medium energy; and blue, high energy X-rays). The numerous point sources are associated with binary star systems. Diffuse X-ray emission in the very central region is due to the hot gas heated probably by shock waves from supernova explosions. The optical image of the Andromeda Galaxy was taken at the National Science Foundation's 0.9-meter telescope on Kitt Peak (Tucson, Arizona) with the NOAO Mosaic CCD camera.

the past and current X-ray facilities and gives access to the archival X-ray data and many source catalogs (Figure 1).

The X-ray universe contains rich structures and traces signatures of violent events associated with the evolution of different cosmic objects. Most astrophysical sources exhibit some level of activity resulting in X-ray radiation and depending on the quality of the instruments we can study those in great detail. Observations made by the Chandra $\mathrm{X}$-ray Observatory and XMM-Newton over almost two decades have tremendously improved our understanding of the X-ray universe. They also brought new scientific questions and expanded the scope of theoretical studies in many areas of astrophysical research.

\section{X-RAY RADIATION}

Most astronomical objects emit X-rays. The fraction of the power emitted in X-rays varies by orders of magnitude between different types of sources and depends on the specific physical characteristics of a source. Some sources are most luminous when seen in X-rays, while they are almost undetectable in other wave bands. X-ray emissions can provide key information and be critical to understanding physical processes behind the nature and evolution of sources. In the vast majority of sources the gravity is responsible for generating directly or indirectly X-rays.

$\mathrm{X}$-ray radiation can indicate high temperatures $\left(>10^{6} \mathrm{~K}\right)$ which are often observed in the interstellar medium of galaxies and intracluster medium of clusters 
of galaxies. Matter surrounding compact objects (black holes or neutron stars) have high temperatures due to the gravitational energy released in the accretion process. Gas can also be heated to high temperatures by shocks generated, for example, by supernova explosions or by mergers of different systems. In some objects nonthermal processes associated with the presence of highly energetic particles (electrons, positrons, protons, and ions) embedded in a magnetic field or starlight emission can dominate the $\mathrm{X}$-ray radiation. The $\mathrm{X}$-ray emission can thus highlight sites of violent and powerful events leading to heating of the gas and acceleration of the particles. This is important in understanding the power, dynamics and evolution of structures in the universe.

\section{COSMIC X-RAY BACKGROUND RADIATION}

Early X-ray observations performed in the 1960's displayed a relatively isotropic (uniform and smooth) radiation over the entire sky. Only a few bright $\mathrm{X}$-ray sources were detected at that time, including Sco-X1 which was the first detected extrasolar X-ray source (Giacconi, Gursky, Paolini, \& Rossi, 1962). The origin of this isotropic radiation, called $\mathrm{X}$-ray Background, intrigued many astronomers. It was not known whether this radiation was characteristic of the X-ray sky and uniformly distributed everywhere, or was composed of many individual sources that could not be resolved. The X-ray missions in the 1980s and 1990s (e.g., Einstein, ROSAT) detected stars, neutron stars, binaries, supernovae, galaxies, quasars etc, and compiled first X-ray source catalogs. These sources contributed their radiation to the X-ray background and active galactic nuclei were identified as the dominant source population. However, they did not account for the entire emission and resolving the remaining $\sim 30 \%$ of the $\mathrm{X}$-ray background radiation required observations with modern, high sensitivity and high angular resolution telescopes.

The Chandra X-ray Observatory was launched on July 23, 1999. The Chandra telescope contains the highest angular resolution X-ray mirrors to date. It is capable of resolving X-ray sources that are less than 1 arcsec apart. This angular resolution is significantly better than other currently operating X-ray missions, such as XMM-Newton ${ }^{2}$ or NuStar. ${ }^{3}$ The Chandra

\footnotetext{
2 http://www.cosmos.esa.int/web/xmm-newton

3 https://www.nasa.gov/mission_pages/nustar/main/index.html
}

observations revolutionized studies of the X-ray universe and displayed complex X-ray morphology of for example supernova remnants, star clusters, galaxies and clusters of galaxies. XMM-Newton has the highest effective area of the current X-ray telescopes and is best for low and high resolution spectroscopy observations of relatively bright sources. NuStar is the focusing telescope operating at the energies $>10 \mathrm{keV}$ (higher than the Chandra and XMM-Newton energy bands) allowing for spectral and temporal studies at these energies.

Chandra supplied the required technology for studying the X-ray background. The density of X-ray sources increases with the decreasing flux. Resolving the X-ray background into sources required detection of sources at the faintest end $\left(<10^{-15} \mathrm{erg} / \mathrm{s}\right)$ of the source density distribution. A few very long Chandra observations of small sections of the sky, so-called deep fields, detected thousands of faint sources that were identified with distant galaxies and quasars (Giacconi et al., 2001; Mushotzky, Cowie, Barger, \& Arnaud, 2000). The integrated contributions from these sources accounted for more than $90 \%$ of the X-ray background radiation observed by the earlier X-ray missions.

The Chandra Deep Surveys obtained amazing sensitivity limits (Lehmer et al., 2012; Xue et al., 2011; for review see Brandt \& Hasinger, 2005) and detected thousands of sources. The majority of these sources were sampling different classes of active galactic nuclei and were detected out to the redshifts exceeding $z>4$. There were also other types of sources such as a new class of X-ray bright, optically normal galaxies, starburst galaxies and stars. These observations indicated that a variety of astrophysical objects can be found in the X-ray universe. They improved our knowledge of active galactic nuclei evolution and growth of supermassive black holes. They increased the number of known active galactic nuclei at high redshift and allowed for studies of cosmic star formation in distant galaxies (Figure 2).

\section{RELATIVISTIC JETS IN CHANDRA OBSERVATIONS}

On August 14, 1999, the Chandra X-ray Observatory pointed at quasar PKS 0637-752, a calibration target, to focus the telescope. This observation brought an exciting discovery. The quasar, which was supposed to be point-like, exhibited 12 arcsec tail (Schwartz 


\section{MONOGRAPH}

Violent universe

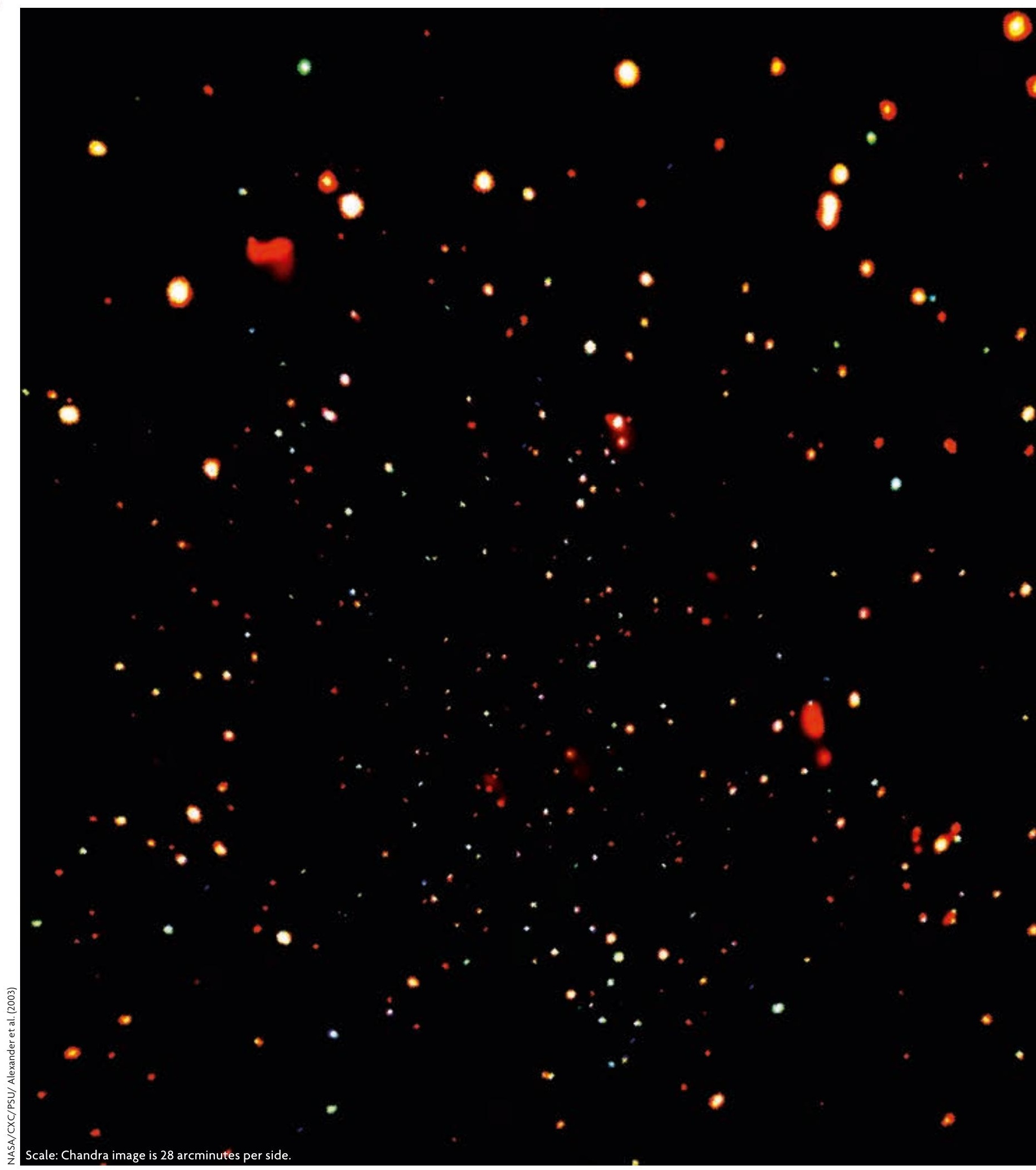

Figure 2. The Chandra X-ray image of the part of the sky located in the Ursa Major constellation (Deep Field North). The deep observation was carried over 23 days and more than $500 \mathrm{X}$-ray sources were detected. The faintest sources produced only one X-ray photon every 4 days. 

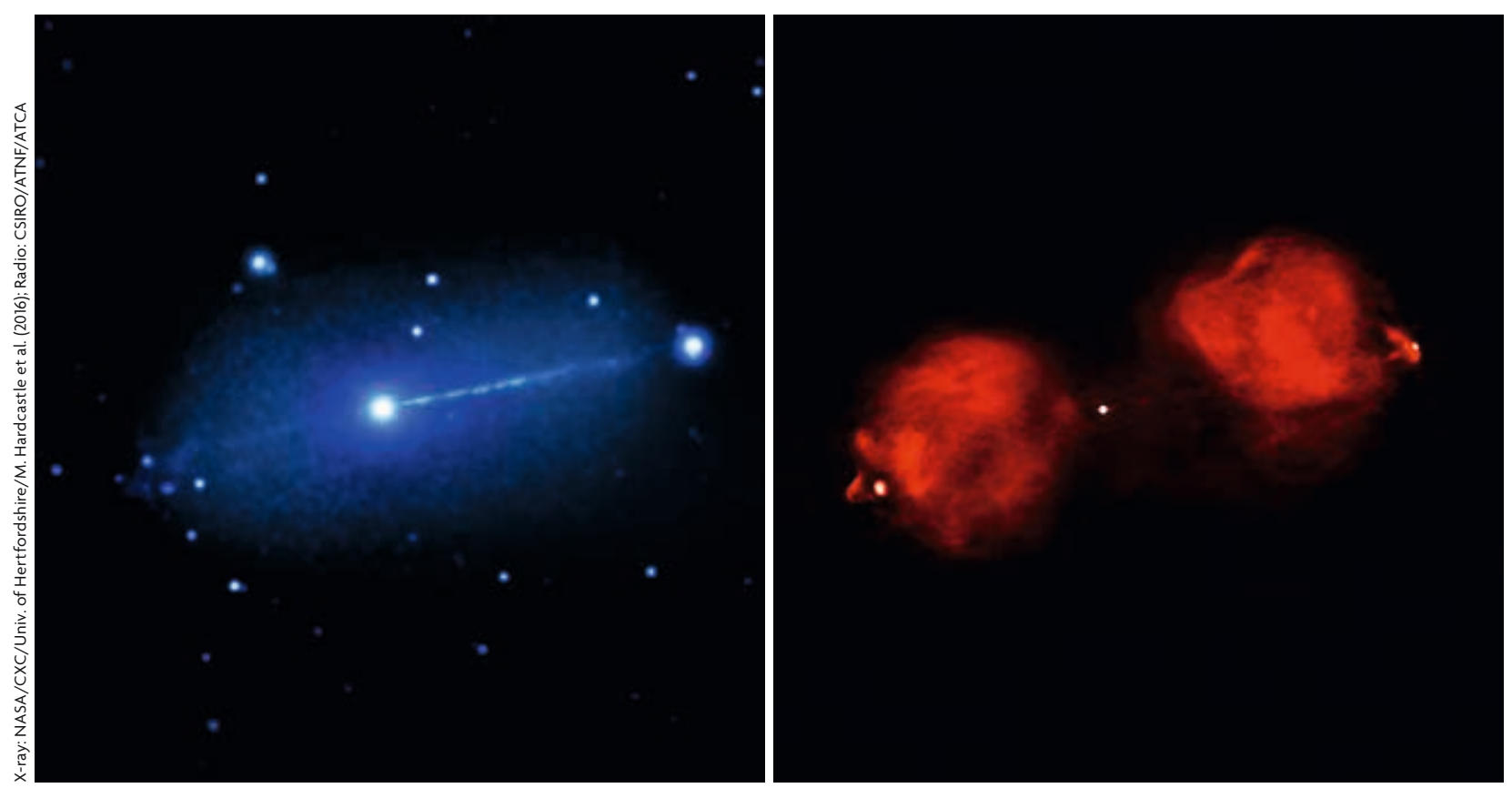

Figure 3. X-ray (left) \& radio (right) images of Pictor A. The Pictor A galaxy has a supermassive black hole at its center, and material falling onto the black hole is driving an enormous beam, or jet, of particles at nearly the speed of light into intergalactic space. These images show X-ray data obtained by Chandra at various times over 15 years and radio data from the Australia Telescope Compact Array. By studying the details of the structure seen in both X-rays and radio waves, scientists seek to gain a deeper understanding of these huge collimated jets.

et al., 2000). It was soon realized that this tail was caused by the X-ray radiation of a relativistic jet that has been known and observed earlier at radio frequencies. The $\mathrm{X}$-ray radiation was a surprise and was found only in the inner straight section of the radio jet. It disappeared at the place where the radio maps show a sharp bend in the jet. This $\mathrm{X}$-ray jet extended beyond the host galaxy and out to about 100 $\mathrm{kpc}$ distance from the quasar and exceeded a total X-ray luminosity of $10^{44} \mathrm{erg} / \mathrm{s}$ (a few percent of the quasar luminosity).

Powerful jets have been associated with black holes in active galactic nuclei. They carry a fraction of energy generated by a black hole to large distances. Jets have been observed at radio frequencies as narrow, elongated features connecting the radio core with large scale radio structures. They can exhibit apparent superluminal motion when observed at very small angles to the line of sight. Only a few jets were detected in X-rays before Chandra. The Einstein High Resolution Imager (HRI) observed a jet from a nearby giant elliptical galaxy, M87 (Schreier, Gorenstein, \& Feigelson, 1982). It also found an X-ray jet associated with the radio galaxy Centaurus-A (Schreier et al., 1979) and a jet from quasar 3C273 (Willingale, 1981). Later on, ROSAT observations provided data for a few more X-ray jets. Unlike Chandra, neither XMM-Newton or Suzaku had the angular resolution to resolve $\mathrm{X}$-ray jets. The X-ray jets are seen as diffuse linear and bending structures with enhancements named knots or hot spots. The jet is often located close to the strong point-like emission of the quasar core, or embedded in the diffuse emission of a host galaxy with a total observed length rarely exceeding about 30 arcsec. Most X-ray jets are faint and their emission is only a small fraction $(<3 \%)$ of a strong core. The high angular resolution and dynamic range observations provided by Chandra has been critical to the detection of X-ray jets. In recent years, many large-scale X-ray jets associated with low power radio galaxies and high power quasars were discovered in Chandra observations (for a review, see Harris \& Krawczynski, 2006).

Several surveys of X-ray jets have been completed to date. These surveys focused on understanding 


\section{MONOGRAPH}

Violent universe

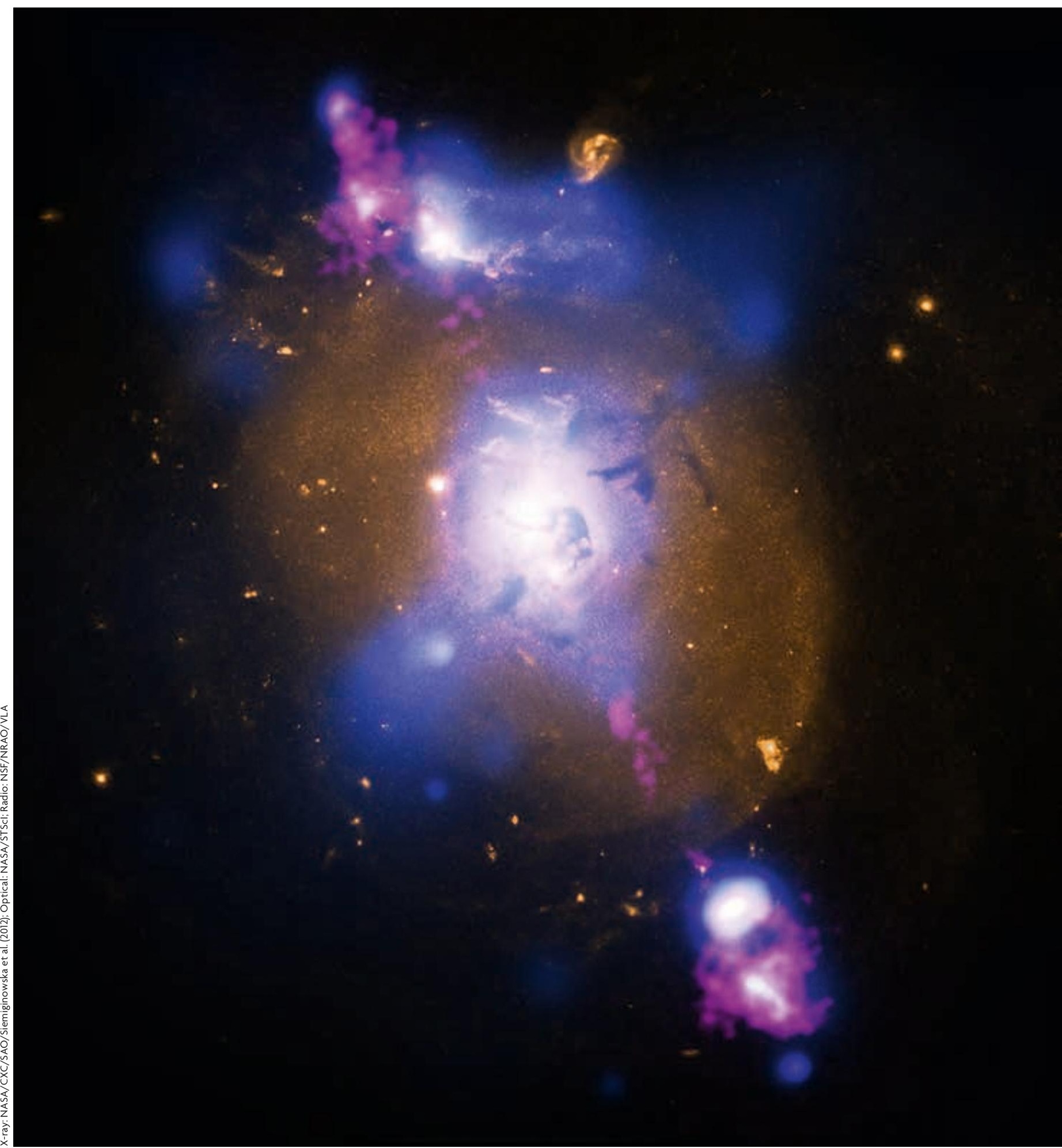

Figure 4. X-ray (blue), optical (yellow) and radio (purple) images of 4C+29.30 galaxy. The intense gravity of a supermassive black hole can be tapped to produce immense power in the form of jets moving at millions of miles per hour. A composite image shows this happening in the galaxy known as $4 C+29.30$ where $X$-rays from Chandra are shown with optical and radio data. The X-rays trace the location of superheated gas around the black hole, which is estimated to weigh 100 million times the mass of our Sun. The optical light image shows the stars in this galaxy. A torus of gas and dust surrounds the black hole and blocks most of the optical light coming from there and the central supermassive black hole is called «hidden» or «buried». 
$\mathrm{X}$-ray emission processes in large scale extragalactic jets. The possible mechanisms include synchrotron emission from highly relativistic particles in the jet or the emission resulting from the inverse Compton process where the jet relativistic particles transfer their energy to lower frequency photons (radio-IR-opticalUV) resulting in the X-ray emission. The source of the photon field could be internal to the jet (synchrotronself-Compton, SSC) or external to the jet. In the large scale jets associated with quasars the Cosmic Microwave Background was considered as the primary source of the photon field (IC/CMB) (Figure 3).

Massaro, Harris, and Cheung (2011) compiled a list of all X-ray jets detected with Chandra. ${ }^{4}$ They used these data to describe statistical properties of all detected X-ray jet features, including knots, hot spots, and diffuse jet emission. These studies indicated a real difference in the radio to X-ray flux ratios between the hotspots and knots in high power radio sources, and no significant difference in the ratios for the knots in low power radio sources and quasar jets. This second result is really interesting, because it suggests that either the knots in both types of jets are due to the synchrotron process, or that the inverse Compton process in quasar knots has very specific conditions resulting in the same flux ratios. The high quality Chandra data indicate the complexity of quasar jets, of the physics responsible for powering jets, and the energy release. These data show that extragalactic jets remain highly relativistic out to megaparsec ${ }^{5}$ distances from their origin. The large scale distances set timescales for the jet activity in the evolving black holes. On the other hand, questions related to the physics of particle acceleration processes at the high distances from the black hole have not been resolved.

While the observational data gathered by Chandra over the past years underlined the significance of jets in evolution of galaxies and clusters of galaxies, there are still many challenging questions about the nature of relativistic jets, energetics, particle content, particle acceleration and emission processes. Both statistical studies of large samples of jets across the entire electromagnetic spectrum as well as deep broad-band observations of individual jets are necessary to make progress on our understanding of jets.

\footnotetext{
4 http://hea-www.harvard.edu/XJET/

51 megaparsec $(\mathrm{Mpc})$ is equivalent to more than three million light years.
}

Knowing jets might bring us closer to understanding the physics of many astrophysical systems across different mass scales.

\section{JETS IMPACT ON THE INTERSTELLAR MEDIUM}

Jets interact with and deposit their energy to the environment and impact evolution of structures, galaxies and clusters of galaxies. The details of jet interactions could be traced in several deep Chandra observations of nearby galaxies. An example of complex morphology resulting from the jet outflow is displayed in an elliptical radio galaxy $4 \mathrm{C}+29.30$ (Figure 4) (Siemiginowska et al., 2012; Sobolewska et al., 2012). The jet and the associated radio emission extending out to the outskirts of this galaxy with associated enhanced optical emission located at a similar distance. The filamentary X-ray emission traces the jet and stretches beyond the galaxy. The bright $\mathrm{X}$-rays to the north overlap with the optical emission regions.

This deep X-ray image provides energetics of the system with much improved detail over the earlier studies in radio and optical bands. The brightest X-ray emission features consist of a mixture of thermal and nonthermal radiation, characterized by a variety of temperatures, with potential differences in metal composition across the galaxy. A significant fraction of the jet energy goes into heating the surrounding gas via weak shocks. Only a small amount of jet power is needed to accelerate clouds of colder material and expel them out of the galaxy. The X-ray radiation from the nucleus of the galaxy is strong but the nucleus is also heavily obscured by a significant amount of matter in-falling onto the center. This infall may be related to feeding the nucleus and triggering the jet activity. The Chandra results for this galaxy support the idea of a feedback between the central supermassive black hole and the environment, which is critical to the evolution of galaxies. Details of the feedback process are still not well understood (Figure 4).

\section{X-RAY EMISSION FROM CLUSTERS OF GALAXIES}

Galaxy clusters form the most massive bound structures in the universe and trace concentrations of dark matter. The volume between galaxies in a cluster is filled with hot medium that can be studied 
in X-rays. Chandra and XMM-Newton observations of clusters show a complex morphology of X-rayemitting gas with cavities, ripples and shocks on many scales. These features represent remnants of the active events in the evolution of a cluster. Signatures of active galactic nuclei outbursts and mergers are often imprinted in the hot medium and can be used to discern the past violent events. For example, «ripples» in the Perseus and other clusters resemble the ripples on the pool of water after a drop of a stone. They have been interpreted as echos from the outburst event more than million years ago. Bubbles and cavities of different sizes and locations in clusters provide information about the total energy released in such events. They also have been used to analyze the energy deposited into the environment and informed about the radio-mode feedback described in the previous section governing the evolution of structures.

Studies of X-ray clusters significantly contributed to modern cosmology (for a review see Allen, Evrard, \& Mantz, 2011). High quality data for a large number of clusters collected by Chandra and XMM-Newton allowed for studies of scaling relations (such as the evolution of mass function, mass and temperature relations, baryon mass fraction, or cluster sizes) important for testing different cosmological models (e.g., flat or evolving universe with different cosmological constant parameter). Addition of X-ray data to scaling relations improved the statistical uncertainties on the measured parameters (Figures 5 and 6).

\section{FUTURE}

The last decade of X-ray observations with Chandra and XMM-Newton indicated that the X-ray emission is common and can be detected in all types of cosmic sources. X-rays are often the key to investigation of energetics, dynamics and evolution of many objects and, thanks to these observations, our knowledge of how the universe works has significantly improved. However, many important science questions remain unanswered and require future investigations. The next big step will be European Space Agency's Athena (Advanced Telescope for High Energy Astrophysics), ${ }^{6}$ due for launch in 2028 , which will offer transformational capabilities in sensitive imaging, timing and spatially-resolved high-resolution spectroscopy in the X-ray band.

New technologies are necessary to improve the angular resolution of X-ray images in the future. They

6 http://www.the-athena-x-ray-observatory.eu/

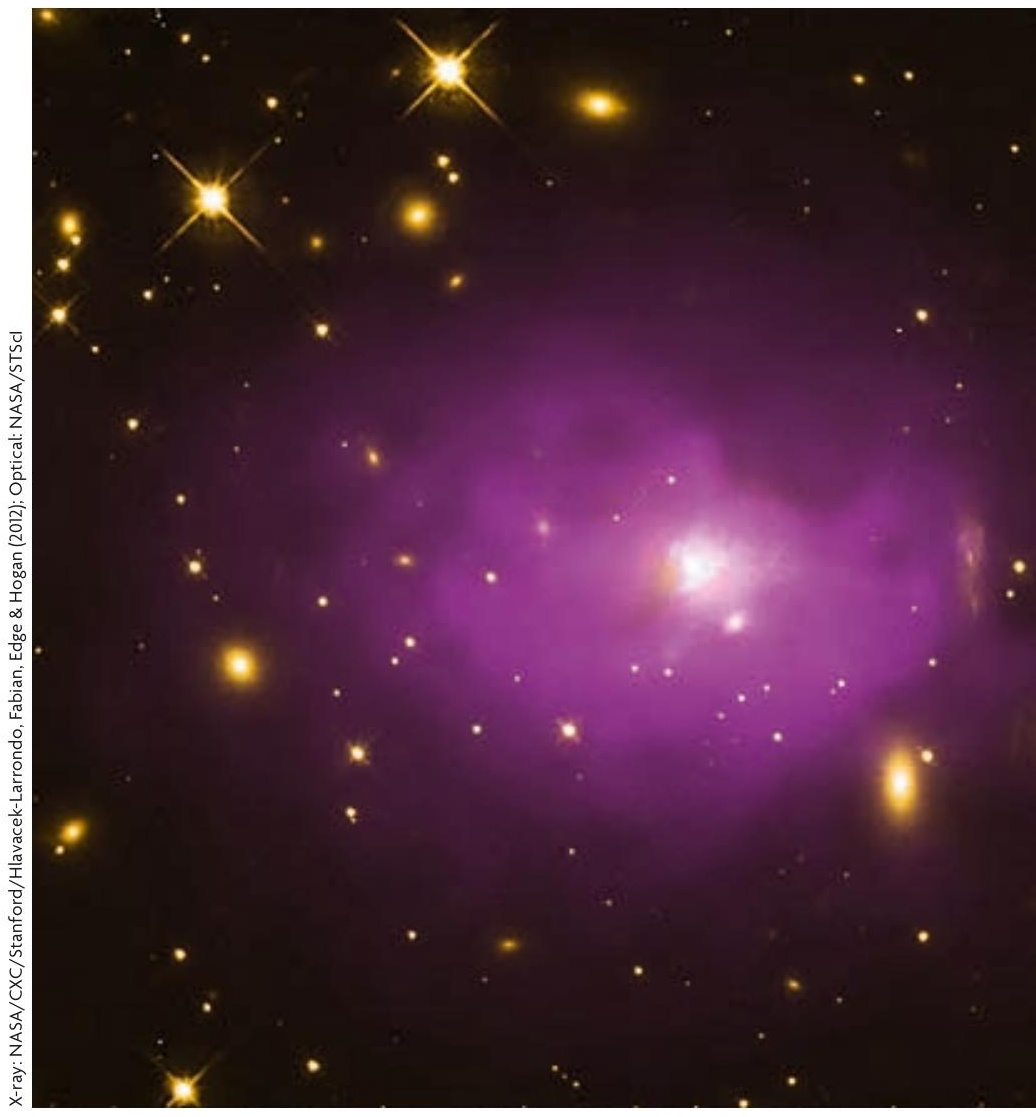

Figure 5. X-ray (purple) and optical (yellow) images of the cluster of galaxies Abell 2204. The black hole of the central galaxy in this cluster is part of a survey of 18 of the biggest known black holes in the universe. Researchers found that the black holes in the survey may be about ten times more massive than previously thought. This includes at least ten that could weigh between 10 and 40 billion times the mass of the Sun, making them «ultramassive» black holes.

Figure 6. X-ray (blue), radio (pink) and optical (yellow) images of Hydra A. These images of the Hydra A galaxy cluster show 10-million-degree gas observed by Chandra (blue) and jets of radio emission observed by the Very Large Array (pink). Optical data from the Canada-France-Hawaii telescope and the Digitized Sky Survey shows galaxies in the cluster (yellow). Detailed analysis of the Chandra data shows that the gas located along the direction of the radio jets is enhanced in iron and other metals produced by Type la supernova explosions in the large galaxy at the center of the cluster A powerful outburst from the supermassive black hole then pushed the material outwards, over distances extending for almost 400,000 light years, extending beyond the region shown in this image.

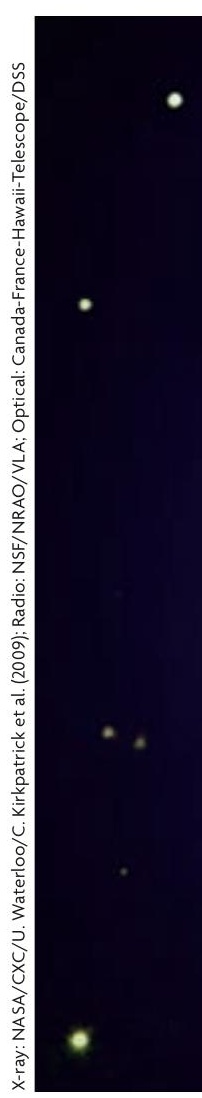


